\title{
A Booking Type Production System as a Collaboration Method for Virtual Enterprises
}

\author{
Y. Nishioka, Y. Fukuda \\ Hosei University, Japan \\ Em: nishioka@k.hosei.ac.jp \\ Y. Kamio \\ Toyo Engineering Corporation, Japan \\ K. Kawashima \\ Mitsui Engineering and Shipbuilding Co.Ltd., Japan
}

Keywords Advanced Planning and Scheduling, Reactive Scheduling, Supply Chain Planning, Reservation System, Virtual Enterprise, Internet

\begin{abstract}
A booking-type production system (BTPS) has a coordination mechanism to synchronize manufacturing processes with customer requirements in the dynamic environment. This paper introduces BTPS, and extends it with Advanced Planning and Scheduling techniques. Some future capabilities of the system in Internet environment are also discussed.
\end{abstract}

\section{INTRODUCTION}

For some manufacturing companies, a booking-type production system (BTPS) can perform as an effective collaboration tool between a manufacturing division and a sales division. While a sales division knows customers' orders and puts them on an online booking chart, it is always available to promise because the booking chart describes the manufacturing division regarding the shop floor capacity and the relevant procurement situations. When BTPS was implemented in early 1990s in Japan, the booking charts were static so that there were some inefficient features. This paper deals with BTPS in dynamic environment. Furthermore, the target of the system is extended for a virtual enterprise that consists of different firms. The key technology for this purpose is re-calculating the booking chart using 
advanced planning and scheduling (APS) algorithms. In addition, web-based technologies such as XML/EDI are also important. This paper illustrates and discusses a framework of a collaboration architecture using BTPS and the relative Internet technologies for competitive virtual enterprises.

The remainder of this paper is organized as follows. In section 2, conventional BTPS is introduced, and then, section 3 shows an extended BTPS, that has an APS module and its capabilities. The main feature of the extended BTPS is dynamic arrangement of the booking chart. Section 4 explains this problem using a simple example. The proposed architecture of our BTPS is illustrated in section 5. Then, in section 6, an implementation example is addressed. Finally, section 7 concludes our approach as a collaboration architecture for virtual enterprises.

\section{BOOKING-TYPE PRODUCTION SYSTEM}

To synchronize manufacturing processes with customer's demands, BTPS uses an online booking-chart (OBC). The OBC is a user-friendly interface to reserve final products, which will be finished in the future. All sales persons can access it from different located offices. In early 1990s, some Japanese manufactures developed computer integrated manufacturing (CIM) systems successfully with BTPS. TOSHIBA Corporation, consumer electrical machine company, is the first to establish the system, in which final products on $\mathrm{OBC}$ are reserved [1,2]. Another industrial example can be shown by Toyoda Machine Works, Ltd., one-of-a-kind mother machine manufacturing company [3]. In this case, the target of reservation is not a final product but manufacturing resources such as machine-hours and person-hours.

Using BTPS, effective manufactures have some important advantages in the market places. First of all, customers can get a quick response of their order receipt with a concrete due date. Since the OBC shows availability to ship the required products, a sales person can enter a new order while he/she answers the due date. Secondly, from a shop floor manager's point of view, BTPS protects ineffective production orders. In other words, customers cannot select alterative dates or products that are not on the OBC. This contributes to increased productivity of the plant. Finally, long-term order reservation by customers contributes more precise demand forecasting, even if these orders might be cancelled in the future.

The business process in BTPS is similar to seat reservation systems in airline companies [4]. In accordance with the customer orders, sales persons reserve items on the OBC after a master production schedule (MPS) is fixed and a corresponding schedule is released. Up to the time a few days from the 
production execution date, orders are arriving one after another. When the reservation date is more than one month from the execution date, the sales person can cancel these orders with no charge. BTPS allows users to cancel their orders within one month if the modification of the order is less than fifteen percent. However, within five days, all of these modification or cancellation are denied. The business process above is a typical example, and there could be many variations for each company's characteristic.

\section{SYSTEM EXTENTION WITH APS}

Generally, production systems should follow each customer's order. To do this, production schedule is usually made after customer's orders arrive. In contrast, BTPS makes schedule before each order can be identified. This implies that, in BTPS, customers have to adjust to the production schedule, whereas conventional systems allow that the production schedule adjusts their customers.

Even if BPTS has many advantages in terms of synchronized manufacturing, this argument is true. Therefore, we try to modify BTPS to a more customer-oriented system. A solution to get this goal will be provided by advanced planning and scheduling (APS) technologies.

Scheduling systems were firstly developed for shop floor managers in deciding which order is started first and so on. Now, a successor of this kind of scheduling systems, so-called APS, has many capabilities. We can identify two important features for BTPS. One is rescheduling capability, which allows shop floor planners to revise the current schedule when some unexpected events occur. Another capability of APS is consideration of inventory level of raw materials, work-in-process inventories, and final products. By this function, APS can manage purchasing information, which the conventional MRP system used to provide.

Considering these capabilities, a benefit of BTPS with APS can be suggested in rearranging the $\mathrm{OBC}$. There are several ways to rearrange the OBC. One is to rearrange regularly, for instance, every week. This is very simple to operate both for a production planner and a sales person. On the other hand, there are many instances of dynamic rearrangement, which is made anytime when sales persons or shop floor planners request.

In general, rearrangement should be done by combination of the two cases. However, the later case is very difficult to achieve without an integrated APS module.

\section{DYNAMIC BOOKING-CHART REARRANGEMENT}


The proposed system framework uses APS to rearrange the booking chart dynamically. This rearranging is affecting customer satisfaction in reserving their preference. If the OBC cannot be modified, many customers are forced to change their requirements when the OBC does not offer appropriate one. Some customer may regret their requirements and may change their mind of ordering. The rearrangement with APS preserves the OBC has most appropriate candidates for forthcoming orders. This is valuable especially in the case that a forecasting demand differs from the real trend of the orders. We found out that this modification increase customer satisfaction by numerical experiments [5].

Using a simple example, benefit of booking chart rearrangement is explained. Figure 1 shows bill of material and process (BOMP) structures of sample products. The product $\mathrm{A}$ is made by a process, which occupies machine $\mathrm{X}$ for 30 minutes per quantity. The figure also shows that, the process requires 2 of $\mathrm{M} 1$ and 10 of $\mathrm{M} 2$ as raw materials. The product $\mathrm{B}$ and the $\mathrm{C}$ are defined in the same way. In this case, the three products share the machine $\mathrm{X}$ and the material M1. This causes competition in BTPS in making OBC.

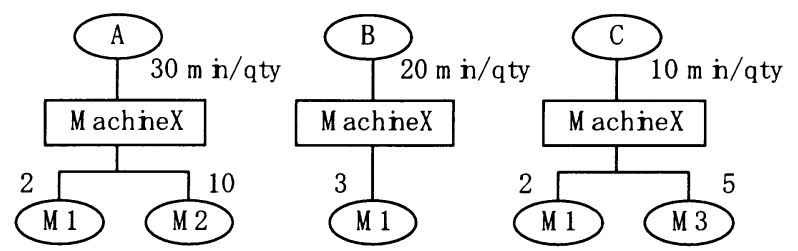

Figure 1: Bill of Material and Process (BOMP) of Sample Products

Table 1 Production availability calculation by resource and material usage.

\begin{tabular}{llllll}
\hline Product & Machine X & M1 & M2 & M3 & Minimum \\
\hline A & 4 & 10 & 3 & --- & 3 \\
\hline B & 6 & 6 & -- & --- & 6 \\
\hline C & 12 & 10 & --- & 12 & 10 \\
\hline Available & 120 & 20 & 30 & 60 & --- \\
\hline
\end{tabular}

BTPS calculates each available number of the final products for OBC. The calculation procedure can be explained in Table 1. When the total availability is given for each resource and material, the number of each product is calculated for each column on the table. The total availability of each product is the minimum number of the corresponding row. The table 1 shows the results of the quantity to make $\mathrm{A}, \mathrm{B}$, and $\mathrm{C}$ are 3,6 , and 10 respectively. 
Because there are resource and material confliction, the available numbers 3, 6 and 10 for the products are not realized at a same time. One feasible solution is 2,2 and 2 for the product A, B and C, respectively. Obviously, if these feasible numbers are fixed on $\mathrm{OBC}$, it restricts flexibility of the shop floor. BTPS with APS makes possible of dynamical rearrangement, so that, if there are particular demand such as 10 of the product $\mathrm{C}$, these orders are accepted after rearranging $\mathrm{OBC}$.

\section{THE SYSTEM ARCHITECTURE}

The system architecture of the proposed system can be shown in Figure 2. This system consists of different modules [5]. One typical module is the booking management module, which releases and revises the booking chart through the network. An order entry system in the booking management module and the OBC accessible from the different places perform cooperatively. These orders are divided into long-term orders and short-term orders, and then stored in the database.

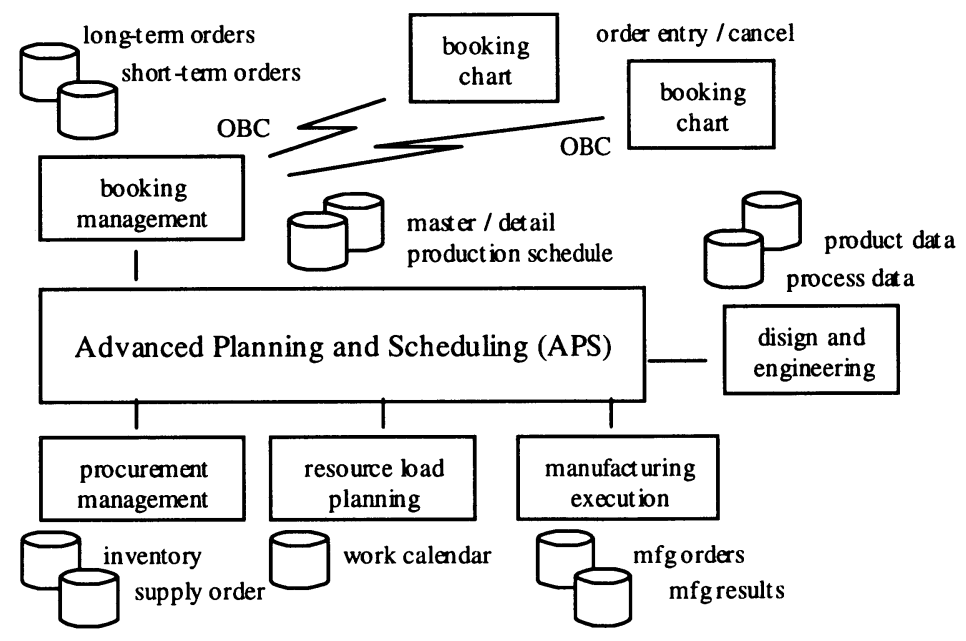

Figure 2: System framework of BTPS [5].

The APS module is located in the central part of the system to calculate an initial schedule using MPS. After that, the APS revises the schedule when predicting gaps on the OBC appear. The result of the scheduling is stored as a detail production schedule, and frequently used to rearrange the ineffective OBC. Around the APS module, there are several modules such as procurement management, resource load planning, manufacturing execution, 
and design and engineering modules. The advantages to integrate BTPS with APS come from these close connections of the modules.

In the current Internet infrastructure, BTPS should change its framework to more effective one. In this situation, sales persons are not only the persons who enter the orders. Various kinds of customers, who might be procurement staffs of a different company, can operate the BTPS through Internet. An example of use cases can be illustrated in Figure 3.

Regarding Internet, OBC should be upgraded to a web-based booking chart (WBC). Using a web browser with some security protection, WBC can be shared by all relevant customers without any investment. As shown in Figure 3, an enterprise needs its suppliers' BTPS to reserve some parts or materials as its planning/scheduling preconditions. Of course, these reservations might be changed. From supplier's point of view, they can prepare the future demand. And more importantly, it is clear that the business speed and occasion are obviously increased by the web-based BTPS.

A virtual enterprise, which consists of different companies, needs a collaboration method against their different interests. BTPS has a capability to coordinate stakeholders using a reservation system. Consequently, a dynamic supply chain will be successfully managed by the extended BTPS.

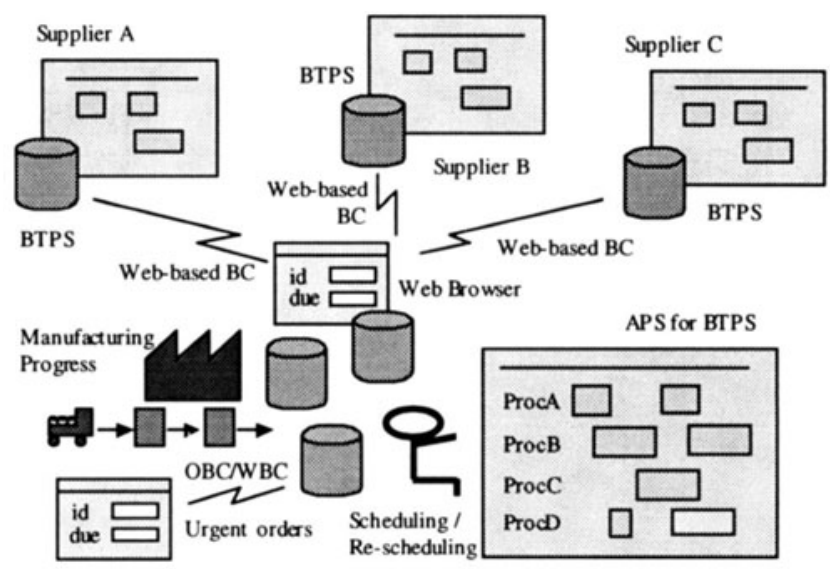

Figure 3: BTPS in Internet environment for VE

\section{IMPLEMENTATION}

We have an APS system named APSTOMIZER [6]. Our APS has a simulation based scheduling logic and penalty propagation re-scheduling logic with a flexible user interface. In order to deal with practical-level constraints, the system keeps tight relationship to Planning and Scheduling 
Language (PSL), which might be a standard of a specification language in all kind of manufactures in Japan [7]. Using APSTOMIZER, we developed an extended BTPS in the Internet infrastructure. Figure 4 shows a Gantt chart of a typical schedule used in the BTPS. The sample manufacture has two product series $A$ and $B$. Each product series has same instances of $A 1$, $\mathrm{A} 2, \mathrm{~B} 1 \mathrm{X}, \mathrm{B} 1 \mathrm{Y}, \mathrm{B} 2 \mathrm{X}$ and $\mathrm{B} 2 \mathrm{Y}$. This scheduling result is used to make $\mathrm{OBC} / \mathrm{WBC}$ information for the BTPS.

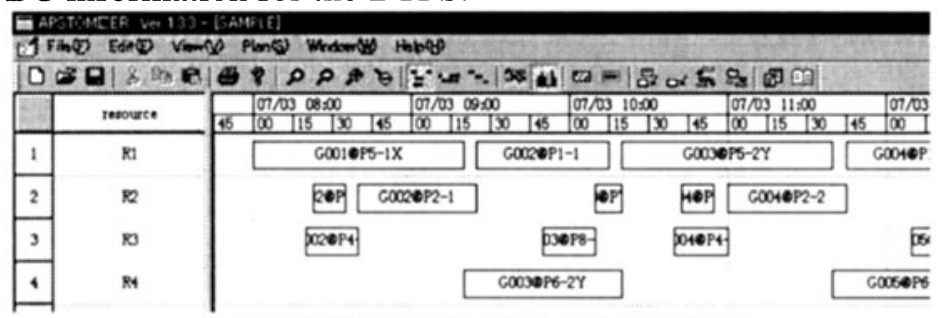

Figure 4: Scheduling result on Gantt chart

When the forecasted demand and the actual demand have a gap, and rearrangement of $\mathrm{OBC} / \mathrm{WBC}$ is required, APSTOMIZER, first, removes all the tasks that are not reserved. Then, APSTOMIZER regenerates new orders according to an up-to-date forecasting. After that, APSTOMIZER reschedules the additional orders, regarding the pre-allocated orders. Finally, $\mathrm{OBC} / \mathrm{WBC}$ is modified according to the new production schedule.

(a) BTPS avaikbility calculated by APST OMIZER

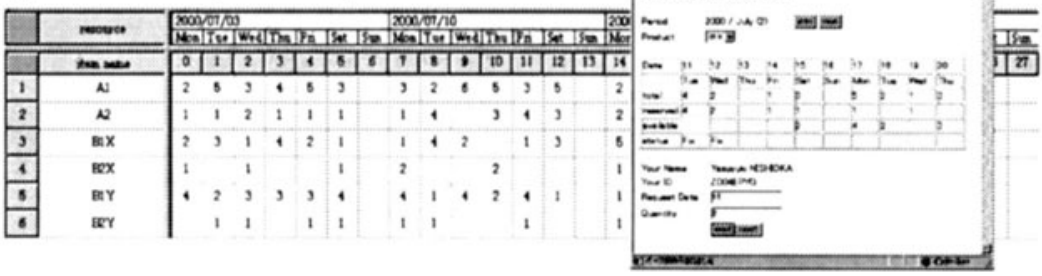

(b) Order entry page for BTPS on the Web

Figure 5: A table of booking chart and a web-based BC

Figure 5 (a) is a table of the available numbers on the booking chart. This also calculated by APSTOMIZER. In this chart, the time bucket is a day, so that, each day has the number of products that will be available to ship. Using this information, the system publishes OBC/WBC to all the sales persons or customers. If BTPS is used only inside of an enterprise, client and server type OBC might be developed on a local network. However, considering supply chain in a virtual enterprise, WBC is necessary. Figure 5 
(b) is an example of web pages provided by the web-based BTPS. In our system, these contents of web-browsers and appropriate web-applications are developed using XML/EDI technologies on a World Wide Web (WWW) server.

\section{CONCLUSION}

A booking-type production system (BTPS) has coordination architecture between a sales division and a manufacturing division. This feature is also valuable between different enterprises, when the system is established on Internet technologies. Using APS capabilities, this paper shows a system framework by modifying a conventional BTPS, in order to make it more customer-oriented. While APS makes rearrangement of the booking chart dynamically, customer satisfaction is obviously increased. Furthermore, a web-based BTPS will provide an effective supply chain in virtual enterprises.

We developed a prototype system of BTPS with APS. The system can be extended for the Internet infrastructure. This feature has a huge impact on virtual enterprise collaboration, especially in the case that members of the VE don't have any close relationship in advance. Even if the user of webbased BTPS belongs to different organizations and different cultures, the system performs well according to the pre-agreed reservation rules.

For future works, we apply this framework to one-of-a-kind production on a virtual enterprise such as ship building manufacturers and $\mathfrak{a}$ plant engineering companies. In this production, partnership between enterprises is much important, so that, a web-based BTPS will take an important role in the virtual enterprise. In addition, many research topics on BTPS such as price discount in reservation, and lot sizing for the booking chart are also our future target.

\section{ACKNOWLEGEMENT}

This research has been carried out under the GLOBEMEN project, one of the IMS International Programs. The authors would like to acknowledge all the members of GLOBEMEN project for variable support.

\section{REFERENCES}

[1] Matsuura J. (1990). CIM in assembly process of information processing machinery industry: A case of Toshiba Ome plant, Automation, 37(8), 29-43 (in Japanese) 
[2] Kuga T. (1994). Production control system for individual order: Load levelling by Toyoda's seta assignment system. Management Systems, 4(1), 20-24 (in Japanese)

[3] Tamura T. and Fujita S. (1995). Designing customer oriented production planning system (COPPS), Int. J. of Production economics, 41, 377-385.

[4] Tamura T., Fujita S. and Kuga T. (1997). The concept and practice of the production seat system, Managerial and decision economics, 18, 101-112.

[5] Nishioka Y. (2000). Coordination Architecture for Supply Chain Management Using A Booking-Type Production System, POM 2000 Proceedings, Sevila Spain.

[6] Nishioka Y. (2000). Production Scheduling Library, http://www.img.k.hosei.ac.jp/pslib/

[7] Nishioka Y. (1999). An Generalized Language of Production Scheduling Problems as a Basis of Supply Chain Management, Proceedings of APIEMS'99, 681-684. 